\title{
A Systematic Review of Interventions to Address Accent-Related Communication Problems in Healthcare
}

\author{
Yulong Gu, PhD, Amee P. Shah, PhD \\ School of Health Sciences, Stockton University, Galloway, NJ
}

Background: Linguistic diversity in terms of speech, accent, and dialect can present a barrier to communication in healthcare. This review synthesizes current evidence on the effectiveness of interventions that target accent- or dialect-related communication problems in healthcare.

Methods: Eligible studies were identified through a search of literature databases in 2018 using medical subject heading (MeSH) terms and additional keywords. Articles were screened for eligibility according to predetermined inclusion criteria. Because of the heterogeneity of the studies, effect data were analyzed using narrative synthesis.

Results: Twenty-six studies ( $n=964$ ) were included, reporting a range of interventions that provided accent modification training, role play or simulated patient consultation training, general language and consultation skills training, web-based training, and cultural competence training. Most studies (24 of the 26 ) indicated some benefits of the accent elements in the interventions based on objective or subjective measures. The key benefits were improvements in speech production, communication competence, and academic/clinical performance, as well as perceptual changes.

Conclusion: Notwithstanding the low research quality and lack of standardized measures in the included studies, this review establishes an evidence base for implementing accent-related communication improvement strategies in healthcare. Large randomized controlled studies would be helpful to strengthen this evidence base.

Keywords: Communication barriers, delivery of health care, education-medical, speech production measurement, systematic review

Address correspondence to Yulong Gu, PhD, School of Health Sciences, Stockton University, 101 Vera King Farris Dr., Galloway, NJ 08205. Tel: (609) 626-3647. Email: yulong.gu@stockton.edu

\section{INTRODUCTION}

The quality of communication in healthcare has a significant impact on patient-clinician relationships and patient satisfaction, ${ }^{1}$ patient safety, ${ }^{2}$ health outcomes, ${ }^{3}$ care team collaboration, ${ }^{4}$ and professional liability. ${ }^{5}$ Communication quality problems related to linguistic diversity in terms of accent and dialect are a growing issue in healthcare given the demographic change in the general population and global migration of clinicians. For instance, the United States is the largest importer of nurses, with approximately 219,000 registered nurses who were trained overseas. ${ }^{6}$ More than one-third of the United Kingdom National Health Service medical workforce was trained overseas, ${ }^{7}$ and onethird of the doctors working in Australia were trained overseas. $^{8}$ In addition, the growing cultural heterogeneity in patient populations resulting from immigration has been noted in the United States and Europe. 9,10 The globalization of the healthcare workforce and patient populations presents a challenge for both clinician-patient communication and clinician-clinician communication. Part of this challenge is to achieve speech clarity and comprehension when speakers with different accents or dialects participate in a verbal conversation. For understanding to occur, the speaker must deliver intelligible speech, and the listener must recognize and understand unfamiliar speech.

According to the American Speech-Language-Hearing Association, an accent is a phonetic trait from a person's original language that is carried over into a second language, while dialect is a broader term that may include pronunciation differences, semantic differences of word choice, and grammatical differences (including sentence word order) that denote regional areas or social groups. ${ }^{11,12}$ Although accent and dialect are natural characteristics of speech, they can present a barrier to effective communication in healthcare where a variety of accents and dialects are present. ${ }^{13,14}$ Immigrant health professionals' and patients' strong accents can reduce speech intelligibility. Also often observed during patient consultations is the use of colloquial expressions associated with a local dialect that are incomprehensible to those unfamiliar with the expressions. Research shows that accented speech slows listeners' processing time. ${ }^{15}$ In the healthcare setting, accents and dialects have been associated with perceptions of lower clinical competency in clinician-clinician communications ${ }^{16}$ and clinicianpatient communications, with shorter consultations, ${ }^{17}$ with 


\section{Boolean \\ Operator}

Search Terms

(accent OR dialect)

AND

(communication OR "communication barriers" OR "clinical communication" OR "communication skills" OR "communication competency" OR "cross-cultural communication" OR "professional communication" OR "culturally and linguistically diverse" OR CALD OR bilingual OR multilingual OR "English as a second language" OR ESL OR "limited English proficiency" OR consultation OR speech OR "clear speech" OR speak OR pronunciation OR intelligibility OR comprehension OR understanding OR perception OR "speech perception" OR stereotype)

AND

(health OR healthcare OR medicine OR medical OR clinic OR clinical OR "health occupations" OR "foreign professional personnel" OR "foreign medical graduates" OR "international medical graduate" OR IMG OR clinician OR physicians OR doctor OR nurses OR nursing OR pharmacists OR pharmacy OR dentists OR dental OR "allied health professional" OR "occupational therapists" OR OT OR "occupational therapy" OR "physical therapists" OR PT OR "physical therapy" OR "speech-language pathologist" OR SP OR SLP OR "speech-language pathology" OR caregivers OR patients OR "healthcare consumers")

AND

("accent modification" OR "accent reduction" OR "accent management" OR "accent adaptation" OR "accent processing" OR "accent identification" OR "accent detection" OR "listening exercise" OR "accent listening" OR "vocal imitation" OR intervention OR therapy OR treatment OR remediation OR program OR training OR education OR teaching OR strategy OR initiative OR support OR technology OR tool)

AND

(evaluate OR assess OR outcome OR results OR measure OR standards OR test OR "English language ability test" OR "English proficiency test" OR screening OR effect OR impact OR efficacy OR cost-effectiveness OR retention OR attrition OR clarity OR feedback)

Note: The search terms in italic font are medical subject heading (MeSH) terms.

CALD, culturally and linguistically diverse; ESL, English as a second language; IMG, international medical graduate; OT, occupational therapy; PT, physical therapy; SLP, speech-language pathology; SP, speech-language pathologist.

lower care quality, ${ }^{18}$ and with less trust for the healthcare provider. ${ }^{19}$

A targeted strategy to address the communication problems in healthcare related to accent and/or dialect is accent modification training programs, often conducted among health students such as medical residents ${ }^{20}$ and nursing students. ${ }^{21,22}$ Another potential strategy, validated in the laboratory setting, focuses on accent adaptation through exposure to systematic variability in accented speech, via either listening exercises ${ }^{23}$ or listening and imitating accented speech. ${ }^{24}$ Other strategies include using tools and technologies to assist with health communication, such as giving patients a printout of their results or a written explanation to aid patient consultation with accented general practitioners. ${ }^{14}$ However, little is known about the impact of these strategies in the healthcare setting. A 2016 systematic review of communication training programs for culturally and linguistically diverse health students concluded that objective evidence of improved skills or performance posttraining is limited. ${ }^{25}$ In this review, we expanded the scope of the 2016 review $^{25}$ to examine interventions that address accent- and dialect-related communication problems in all healthcare contexts, including health education programs and healthcare delivery systems. The intervention subjects include culturally and linguistically diverse health students and professionals (such as immigrant healthcare professionals), professionals who are minorities or for whom English is their second language (ESL), international medical graduates, and international nursing graduates. Our goal was to synthesize the evidence on the effectiveness of interventions that target communication problems related to accent or dialect in healthcare.

\section{METHODS}

To identify scientific evaluations of accent- or dialectrelated interventions in healthcare, we systematically searched and selected relevant studies ${ }^{26,27}$ and included both quantitative and qualitative studies in this review, as findings from all types of studies may contribute to understanding the effectiveness of interventions. ${ }^{28}$ Before undertaking the review, we developed a review protocol according to the Preferred Reporting Items for Systematic Reviews and Meta-Analyses (PRISMA) guideline. ${ }^{29}$ The protocol defined the literature search strategy (Table 1), study eligibility criteria (Table 2), study selection procedure, quality of evidence criteria, and data collection and analysis methods.

\section{Literature Search Strategy}

Eligible studies published between 1990 and 2018 were identified through a search of literature databases using medical subject heading (MeSH) terms and additional search terms relevant to the research topic. The databases searched included MEDLINE (via PubMed), Cochrane Central Register of Controlled Trials (via Ovid), Embase (via Ovid), Cumulative Index to Nursing and Allied Health Literature (via EBSCOhost), PsycINFO (via EBSCOhost), Education Resources Information Center (via EBSCOhost), Linguistics Database (via ProQuest), and ScienceDirect. To retrieve further possible articles, we searched the reference lists in the examined studies and in relevant systematic reviews, conducted a hand search of relevant authors and journals, and searched Google Scholar for grey literature such as unpublished studies and dissertations, conference proceedings, and university websites. 


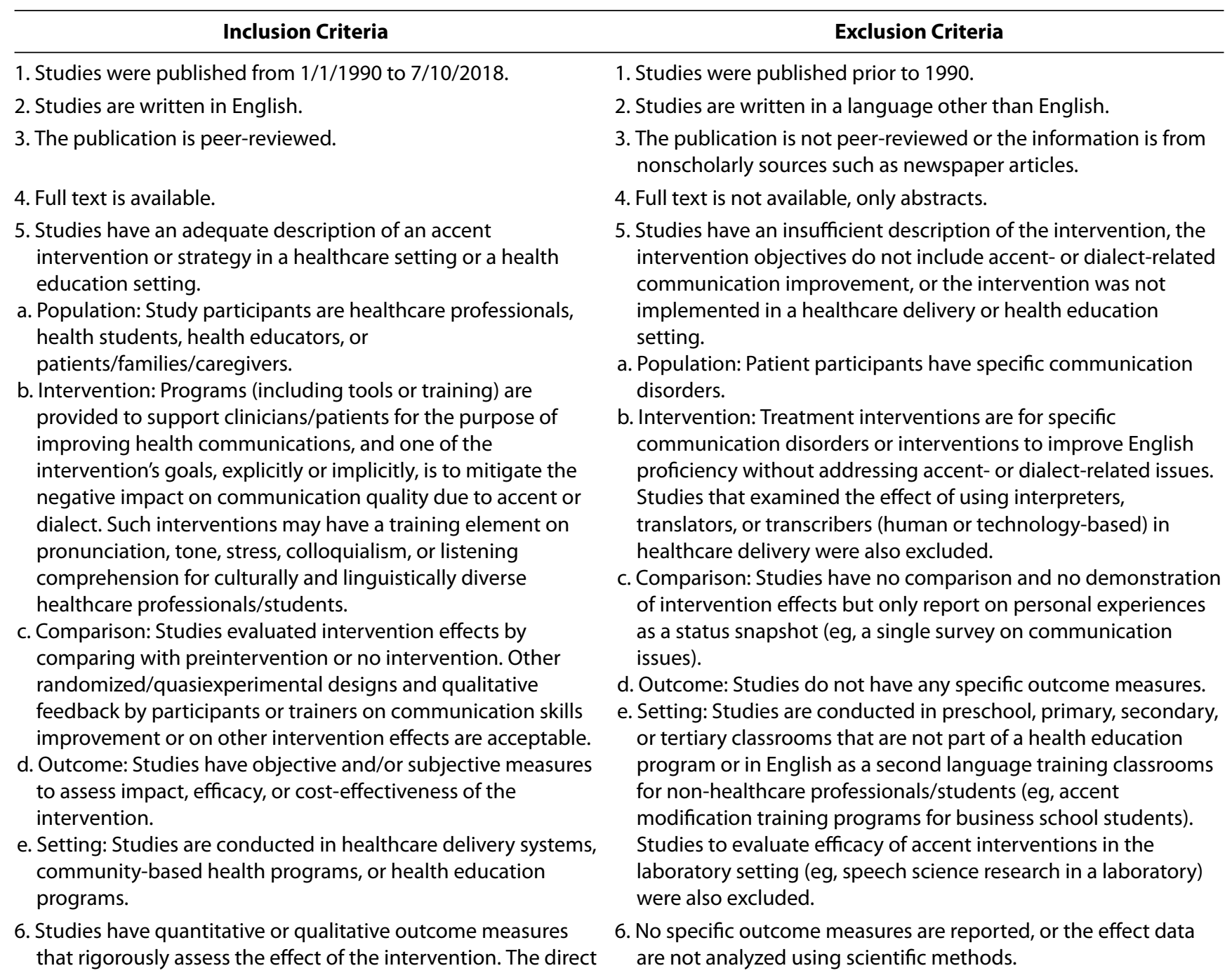
intervention effect on the individual and organization and the indirect effect (eg, on patient outcome) are of interest in this review. The intervention effect in terms of communication quality, clinical performance, health outcome, and cognitive or behavioral change are to be considered.

7. English is the dominant language for communication in the clinical or academic setting.

8. All study designs used to evaluate an individual intervention (eg, randomized controlled trials, other comparative studies, surveys, descriptive studies, etc) were included if they fit the criteria above.
1. Studies were published prior to 1990.

2. Studies are written in a language other than English.

. The publication is not peer-reviewed or the information is from nonscholarly sources such as newspaper articles.

4. Full text is not available, only abstracts.

Studies have an insufficient description of the intervention, the intervention objectives do not include accent- or dialect-related communication improvement, or the intervention was not implemented in a healthcare delivery or health education setting.

Population: Patient participants have specific communication communication disorders or interventions to improve English proficiency without addressing accent- or dialect-related issues. Studies that examined the effect of using interpreters, translators, or transcribers (human or technology-based) in healthcare delivery were also excluded.

of intervention effects but only report on personal experiences as a status snapshot (eg, a single survey on communication issues). or tertiary classrooms that are not part of a health education program or in English as a second language training classrooms for non-healthcare professionals/students (eg, accent modification training programs for business school students). Studies to evaluate efficacy of accent interventions in the were also excluded. are not analyzed using scientific methods.

7. English is not the dominant language for communication in the study setting.

8. Expert opinions and review of studies were excluded.

\section{Study Selection Procedure}

The authors independently reviewed the titles and/or abstracts of all retrieved articles for eligibility according to the study eligibility criteria (Table 2). We discussed and reconciled discrepancies in screening decisions on 5 abstracts; none of the 5 was included in full-text screening. We then retrieved and reviewed the full text of the remaining studies using the same procedure: independent reviews by the authors followed by discussions until the authors agreed upon the selection decisions.

\section{Criteria for the Quality of Evidence}

To assess the validity of included studies, we examined each individual study in terms of the level of evidence, ${ }^{30}$ the quality of evidence, ${ }^{31}$ the overall research quality (considering the research method, reported measures, and study rigor), and the risk of bias. ${ }^{32}$ Taking into account the wide range of research designs and outcome measures used in accent studies, we defined the following criteria based on existing quality appraisal tools and clinical expertise to evaluate the quality of accent intervention studies: 
- High quality: High-level evidence (eg, randomized controlled trial [RCT], cohort, and case-control studies) with low risk of bias and statistical significance tested of objective measures related to communication quality (including flow-on effects such as patient outcome)

- Moderate quality: RCT, cohort, or controlled studies with low risk of bias but with no statistical testing of objective measures (eg, reporting only subjective measures such as qualitative feedback)

- Low quality: Uncontrolled studies (eg, case series) or poorquality cohort control studies with significant limitations in scientific rigor, result generalizability, or reproducibility

\section{Data Collection and Analysis Procedures}

Data from the eligible studies were extracted and recorded in Microsoft Excel. Multiple reports of one study were collated into a single data point. Extracted data items from each study included the following:

- Country where the study was conducted

- Study design

- Study quality evaluated as described in the Criteria for the Quality of Evidence section

- Type and number of intervention participants (health student, healthcare professional, or patient)

- Content, structure, duration, and delivery form of the accent or dialect intervention (only the intervention elements relevant to accent or dialect topics were included)

- Objective (standardized assessment tools such as established language tests and validated questionnaire surveys and nonstandardized measures based on a theoretical framework or on consistent rubrics such as intelligibility test grades assessed by the trainer) and subjective (qualitative feedback and self-rated competence without a rubric) outcome measures (pretest and posttest, if available) relevant to the short- and long-term effects of the intervention

- Study results

We planned both a meta-analysis and a narrative synthesis of the data. However, we found substantial heterogeneity among the studies and a range of nonstandardized measures used to assess the intervention effects. As a result, meta-analysis was infeasible, and the narrative synthesis method was used to analyze the data.

\section{RESULTS}

We reviewed a total of 729 abstracts; 57 studies met the inclusion criteria and underwent full-text review. Among these, 31 studies were excluded (Figure). Of the 26 studies included in this review, 15 were conducted in the United States, 9 in Australia, and 2 in the United Kingdom. One of the 26 studies was a controlled before-after study, 22 were case series, and 3 were case studies.

\section{Characteristics of Accent Interventions}

The studies included in this review described several accent intervention strategies:

- Accent reduction training, often led by a speech-language pathologist (SLP) $21,22,33-38$

- Role play or simulated patient consultation training ${ }^{7,39-48}$

- General language and consultation skill training ${ }^{49-52}$

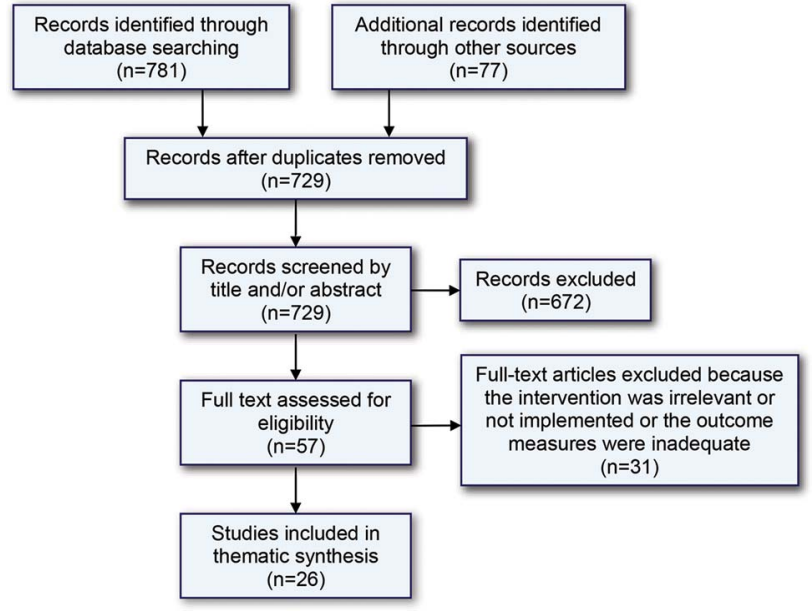

Figure. Flowchart of search and selection of studies for review.

- Internet-based education ${ }^{53,54}$

- Cultural competence training ${ }^{55}$

While each study involved communication skills training, all had distinctive program content, structure, and duration (ranging from 1 session to 2 semesters). Only 3 studies mentioned that the interventions were based on established accent interventions; one study ${ }^{33}$ was based on the Compton Pronouncing English as a Second Language Program, ${ }^{56}$ another study ${ }^{37}$ used the Comprehensive Assessment of Accentedness and Intelligibility model and method, ${ }^{57}$ and the third study had expanded the trainer's own accent reduction course regularly taught at a university. ${ }^{38,58} \mathrm{~A}$ range of linguistic elements related to accent or dialect were taught in the included studies: segmental production (consonants and vowels), phonological and prosodic rules, colloquial/idiom/dialect language, vocabulary and medical terminology, grammatical features, nonverbal/body language/pragmatic features to supplement speech understanding, auditory discrimination, and listening skills. Many studies targeted specific populations such as undergraduate and postgraduate culturally and linguistically diverse health students, international medical graduates, and culturally and linguistically diverse/ESL practicing health professionals. In the delivery of accent interventions, the majority of included studies $(n=17,65 \%)$ engaged a language expert, such as an SLP or English teacher. Among these 17 programs, 9 (often accent modification training programs) employed a language expert as the sole instructor, $21,33,34,36-38,45,49,50$ while 8 used a multidisciplinary teaching team that included both a language teacher and a clinical teacher. ${ }^{7,22,41-44,47,48}$ Study details are provided in Table 3.

\section{Synthesis of Study Results}

Participant Characteristics. A total of 964 participants were involved in 23 studies; the other 3 studies did not specify class size/participant number but were included in this review because they reported relevant intervention descriptions, evaluation methods, and results. More than three-fourths of the study participants were health 
Table 3. Summary of Accent- and Dialect-Related Intervention Elements and the Intervention Effects in the Included Studies

\begin{tabular}{|c|c|c|c|c|c|c|c|c|c|c|}
\hline \multirow[b]{2}{*}{ Study } & \multirow{2}{*}{$\begin{array}{l}\text { Study } \\
\text { Quality }\end{array}$} & \multicolumn{2}{|c|}{ Participants } & \multicolumn{2}{|c|}{ Accent Intervention } & \multicolumn{2}{|c|}{ Objective Instruments } & \multicolumn{2}{|c|}{ Subjective Measures } & \multirow[b]{2}{*}{ Effect Results } \\
\hline & & Type & $\mathbf{n}$ & Content/Structure & Duration & Pretest & Posttest & Pretest & Posttest & \\
\hline $\begin{array}{l}\text { Yahes and } \\
\text { Dunn, } \\
1996^{48}\end{array}$ & Low & INGs & 30 & $\begin{array}{l}\text { Weekly 2-hour sessions } \\
\text { led by SLP and nursing } \\
\text { educator. Content: } \\
\text { pronunciation, accent } \\
\text { remediation, } \\
\text { phonetics. }\end{array}$ & 12 weeks & None & None & None & $\begin{array}{l}\text { Trainee feedback } \\
\text { Clinical supervisor } \\
\text { feedback }\end{array}$ & $\begin{array}{l}\text { Anecdotal comments on } \\
\text { increased job } \\
\text { satisfaction, fewer } \\
\text { communication- } \\
\text { related complaints } \\
\text { from physicians and } \\
\text { staff, improved } \\
\text { collegiality among } \\
\text { staff, and decreased } \\
\text { overall number of } \\
\text { incident reports. }\end{array}$ \\
\hline $\begin{array}{l}\text { Chur- } \\
\quad \text { Hansen } \\
\text { and } \\
\text { Barrett, } \\
1996^{51}\end{array}$ & Low & $\begin{array}{l}\text { ESL } \\
\text { medical } \\
\text { students }\end{array}$ & 44 & $\begin{array}{l}\text { Elective supplementary } \\
\text { course on colloquial } \\
\text { expressions. Structure: } \\
2 \text { tutorials }+ \\
\text { assignment to collect } \\
\text { colloquial expression } \\
\text { examples. Topic: } \\
\text { colloquialism. }\end{array}$ & 1 semester & None & None & None & $\begin{array}{l}\text { Student course } \\
\text { evaluation } \\
\text { questionnaire }\end{array}$ & $\begin{array}{l}\text { Students reported they } \\
\text { learned informal } \\
\text { language. }\end{array}$ \\
\hline $\begin{array}{l}\text { Chur- } \\
\text { Hansen, } \\
1999^{52}\end{array}$ & Low & $\begin{array}{l}\text { ESL } \\
\text { medical } \\
\text { students }\end{array}$ & 87 & $\begin{array}{l}\text { Part of weekly elective } \\
\text { supplementary } \\
\text { tutorials adjunct to } \\
\text { Doctor, Patient and } \\
\text { Society course. Accent } \\
\text { topics: vocabulary and } \\
\text { colloquial language. }\end{array}$ & 2 semesters & None & $\begin{array}{l}\text { Doctor, } \\
\text { Patient } \\
\text { and } \\
\text { Society } \\
\text { course } \\
\text { grades }\end{array}$ & None & Student feedback & $\begin{array}{l}\text { No conclusive benefit or } \\
\text { harm. Tutorial } \\
\text { attendees performed } \\
\text { less well than } \\
\text { nonattendees in the } \\
\text { course, but the author } \\
\text { stated, "without the } \\
\text { program, the disparity } \\
\text { in performance may } \\
\text { have been much } \\
\text { greater and the fail } \\
\text { rates far more } \\
\text { substantial." Students } \\
\text { reported the course as } \\
\text { useful teaching } \\
\text { support. }\end{array}$ \\
\hline
\end{tabular}


Table 3. Continued

\begin{tabular}{|c|c|c|c|c|c|c|c|c|c|c|}
\hline \multirow[b]{2}{*}{ Study } & \multirow{2}{*}{$\begin{array}{l}\text { Study } \\
\text { Quality }\end{array}$} & \multicolumn{2}{|c|}{ Participants } & \multicolumn{2}{|c|}{ Accent Intervention } & \multicolumn{2}{|c|}{ Objective Instruments } & \multicolumn{2}{|c|}{ Subjective Measures } & \multirow[b]{2}{*}{ Effect Results } \\
\hline & & Type & $\mathbf{n}$ & Content/Structure & Duration & Pretest & Posttest & Pretest & Posttest & \\
\hline $\begin{array}{l}\text { Symes et al, } \\
2002^{22}\end{array}$ & Low & $\begin{array}{l}\text { Nursing } \\
\text { students }\end{array}$ & 32 & $\begin{array}{l}\text { Part of a required } \\
\text { 3-credit course, } \\
\text { Student Success } \\
\text { Program, led by an } \\
\text { SLP and a nursing } \\
\text { teacher; 15-hour } \\
\text { accent modification } \\
\text { + } 2 \text { 4-hour periods of } \\
\text { nurse shadowing + } 4 \\
\text { oral presentations. } \\
\text { Topics: medical and } \\
\text { general vocabulary. }\end{array}$ & 2 semesters & $\begin{array}{l}\text { Nurse Entrance } \\
\text { Test reading } \\
\text { comprehen- } \\
\text { sion } \\
\text { test }\end{array}$ & $\begin{array}{l}\text { Student } \\
\text { retention rates } \\
\text { Academic and } \\
\text { clinical pass } \\
\text { rates }\end{array}$ & None & $\begin{array}{l}\text { Trainee feedback } \\
\text { Faculty feedback }\end{array}$ & $\begin{array}{l}\text { Some students } \\
\text { reported more } \\
\text { confidence about } \\
\text { talking to patients } \\
\text { and staff as a result } \\
\text { of the course. }\end{array}$ \\
\hline $\begin{array}{l}\text { Bosher and } \\
\text { Smalkoski, } \\
2002^{39}\end{array}$ & Low & $\begin{array}{l}\text { ESL health } \\
\text { students }\end{array}$ & 18 & $\begin{array}{l}\text { Part of a required } \\
\text { 2-credit course that } \\
\text { meets once a week } \\
\text { for } 2 \text { hours. Course } \\
\text { structure: lecture, } \\
\text { discussion, reading, } \\
\text { journal entry, } \\
\text { student } \\
\text { presentation, 9-10 } \\
\text { role plays per } \\
\text { student. Topic: } \\
\text { paralinguistic } \\
\text { features of } \\
\text { communication (eg, } \\
\text { tone, volume, and } \\
\text { rate of speech). }\end{array}$ & 14 weeks & $\begin{array}{l}\text { Combined } \\
\text { English } \\
\text { Language } \\
\text { Skills } \\
\text { Assessment + } \\
\text { Comprehen- } \\
\text { sive English } \\
\text { Language Test } \\
\text { or University of } \\
\text { Michigan } \\
\text { English } \\
\text { placement test } \\
\text { or University of } \\
\text { Michigan } \\
\text { English } \\
\text { Language } \\
\text { Proficiency test }\end{array}$ & $\begin{array}{l}3 \text { instructor- } \\
\text { graded } \\
\text { role-play } \\
\text { scenarios } \\
\text { Final written } \\
\text { exam }\end{array}$ & $\begin{array}{l}\text { Student } \\
\text { questionnaire } \\
\text { (needs } \\
\text { assessment) }\end{array}$ & $\begin{array}{l}\text { Students' course } \\
\text { evaluation } \\
\text { surveys } \\
\text { Anecdotal } \\
\text { comments by } \\
\text { instructor and } \\
\text { students }\end{array}$ & $\begin{array}{l}\text { Anecdotal student } \\
\text { success stories } \\
\text { about improved } \\
\text { communication } \\
\text { skills. Positive } \\
\text { responses on } \\
\text { course usefulness, } \\
\text { particularly role } \\
\text { plays. }\end{array}$ \\
\hline $\begin{array}{l}\text { Guhde, } \\
2003^{35}\end{array}$ & Low & $\begin{array}{l}\text { ESL } \\
\text { nursing } \\
\text { student }\end{array}$ & 1 & $\begin{array}{l}\text { Weekly 1-hour } \\
\text { one-on-one tutoring } \\
\text { by a nurse tutor. } \\
\text { Topics: listening, } \\
\text { speaking and } \\
\text { pronunciation, and } \\
\text { medical terminology. }\end{array}$ & 10 weeks & $\begin{array}{l}\text { Listening and } \\
\text { note-taking } \\
\text { test }\end{array}$ & $\begin{array}{l}\text { Listening and } \\
\text { note-taking } \\
\text { test }\end{array}$ & None & Trainee feedback & $\begin{array}{l}\text { Increased accuracy in } \\
\text { note-taking. }\end{array}$ \\
\hline
\end{tabular}




\begin{tabular}{|c|c|c|c|c|c|c|c|c|c|c|}
\hline \multirow[b]{2}{*}{ Study } & \multirow{2}{*}{$\begin{array}{l}\text { Study } \\
\text { Quality }\end{array}$} & \multicolumn{2}{|c|}{ Participants } & \multicolumn{2}{|c|}{ Accent Intervention } & \multicolumn{2}{|c|}{ Objective Instruments } & \multicolumn{2}{|c|}{ Subjective Measures } & \multirow[b]{2}{*}{ Effect Results } \\
\hline & & Type & $\mathbf{n}$ & Content/Structure & Duration & Pretest & Posttest & Pretest & Posttest & \\
\hline $\begin{array}{l}\text { Satter et al, } \\
2005^{55}\end{array}$ & Low & $\begin{array}{l}\text { Telephone } \\
\text { survey } \\
\text { inter- } \\
\text { viewers }\end{array}$ & $\mathrm{N} / \mathrm{A}$ & $\begin{array}{l}\text { Training materials + } \\
\text { in-person training. } \\
\text { Topics: American } \\
\text { Indian and Alaska } \\
\text { Natives' cultural and } \\
\text { linguistic issues; } \\
\text { reservation dialect } \\
\text { English, long pauses, } \\
\text { slow speaking, } \\
\text { "walking on words," } \\
\text { and tribal heritage. }\end{array}$ & 1 session & None & $\begin{array}{l}\text { California Health } \\
\text { Interview } \\
\text { Survey } \\
\text { response rate }\end{array}$ & None & $\begin{array}{l}\text { Trainee feedback } \\
\text { Trainer observation }\end{array}$ & $\begin{array}{l}\text { The health survey } \\
\text { response rate in } \\
\text { the target } \\
\text { population is high; } \\
\text { trainees reported } \\
\text { training was } \\
\text { helpful; trainer } \\
\text { observed that } \\
\text { trainees felt } \\
\text { sensitive, } \\
\text { connected, and } \\
\text { responsible to the } \\
\text { community and } \\
\text { were motivated to } \\
\text { do their best. }\end{array}$ \\
\hline $\begin{array}{l}\text { San Miguel } \\
\text { et al, } \\
2006^{45}\end{array}$ & Low & $\begin{array}{l}\text { ESL } \\
\text { nursing } \\
\text { students }\end{array}$ & 15 & $\begin{array}{l}\text { 4-hour sessions per day } \\
\text { for a total of } 20 \text { hours } \\
\text { led by a language } \\
\text { teacher. Topics: } \\
\text { small-talk, formulaic } \\
\text { expressions, } \\
\text { vocabulary, } \\
\text { grammar, and } \\
\text { colloquial } \\
\text { language/switching } \\
\text { register. }\end{array}$ & 5 weeks & None & $\begin{array}{l}\text { Students' clinical } \\
\text { grades at the } \\
\text { end of the next } \\
\text { clinical } \\
\text { placement }\end{array}$ & $\begin{array}{l}\text { Student focus } \\
\text { group on } \\
\text { needs } \\
\text { Facilitators' } \\
\text { written } \\
\text { comments on } \\
\text { students' } \\
\text { clinical } \\
\text { performance }\end{array}$ & $\begin{array}{l}\text { Student written } \\
\quad \text { feedback survey } \\
\text { Student focus group } \\
\text { Facilitator focus } \\
\quad \text { group and } \\
\text { interview }\end{array}$ & $\begin{array}{l}\text { Improved } \\
\text { communication } \\
\text { skills and increased } \\
\text { confidence, } \\
\text { resulting in a more } \\
\text { positive clinical } \\
\text { experience for the } \\
\text { majority of } \\
\text { students. }\end{array}$ \\
\hline $\begin{array}{l}\text { Parkhurst, } \\
2007^{44}\end{array}$ & Low & $\begin{array}{l}\text { ESL phar- } \\
\quad \text { macy } \\
\text { students }\end{array}$ & 25 & $\begin{array}{l}\text { 3-credit elective course. } \\
\text { Structure: role plays, } \\
\text { group practice, and } \\
\text { presentations. } \\
\text { Content: } \\
\text { pronunciation, stress } \\
\text { and intonation, } \\
\text { register, grammar, } \\
\text { medical terminology, } \\
\text { and listening } \\
\text { comprehension. }\end{array}$ & 1 semester & $\begin{array}{l}\text { Michigan Test of } \\
\text { English } \\
\text { Proficiency } \\
\text { Oral summary } \\
\text { examination } \\
\text { Class oral } \\
\text { presentation }\end{array}$ & $\begin{array}{l}\text { Exit examination } \\
\text { using a 4-level } \\
\text { holistic } \\
\text { assessment } \\
\text { rubric. } \\
\text { Students were } \\
\text { graded by } 2 \\
\text { pharmacy } \\
\text { faculty } \\
\text { members. }\end{array}$ & None & $\begin{array}{l}\text { Students' course } \\
\text { evaluation form }\end{array}$ & $\begin{array}{l}\text { Improved oral skills } \\
\text { (eg, intelligibility, } \\
\text { stress, intonation, } \\
\text { and vocabulary) } \\
\text { and listening } \\
\text { comprehension. }\end{array}$ \\
\hline
\end{tabular}


Table 3. Continued

\begin{tabular}{|c|c|c|c|c|c|c|c|c|c|c|}
\hline \multirow[b]{2}{*}{ Study } & \multirow{2}{*}{$\begin{array}{l}\text { Study } \\
\text { Quality }\end{array}$} & \multicolumn{2}{|c|}{ Participants } & \multicolumn{2}{|c|}{ Accent Intervention } & \multicolumn{2}{|c|}{ Objective Instruments } & \multicolumn{2}{|c|}{ Subjective Measures } & \multirow[b]{2}{*}{ Effect Results } \\
\hline & & Type & $\mathbf{n}$ & Content/Structure & Duration & Pretest & Posttest & Pretest & Posttest & \\
\hline $\begin{array}{c}\text { Seibold et al, } \\
2007^{46}\end{array}$ & Low & $\begin{array}{l}\text { CALD } \\
\text { nursing } \\
\text { students }\end{array}$ & 20 & $\begin{array}{l}\text { Semester 1: fortnightly } \\
\text { group meetings; } \\
\text { Semester 2: group and } \\
\text { individual meetings } \\
\text { with academic } \\
\text { mentors. Topics: } \\
\text { colloquial language } \\
\text { and pronunciation. }\end{array}$ & 2 semesters & None & None & $\begin{array}{l}\text { Course entry } \\
\text { questionnaire } \\
\text { (needs } \\
\text { assessment) }\end{array}$ & $\begin{array}{l}\text { Trainee focus group } \\
\text { Trainee exit } \\
\text { questionnaire } \\
\text { Trainee feedback } \\
\text { questionnaire } \\
\text { (3 months } \\
\text { post-program) }\end{array}$ & $\begin{array}{l}\text { The course helped } \\
\text { develop oral and } \\
\text { written } \\
\text { communication } \\
\text { skills. }\end{array}$ \\
\hline $\begin{array}{l}\text { Chiang and } \\
\text { Crick- } \\
\text { more, } \\
2009^{41}\end{array}$ & Low & $\begin{array}{l}\text { CALD } \\
\text { nursing } \\
\text { students } \\
\text { (post- } \\
\text { gradu- } \\
\text { ate) }\end{array}$ & $\mathrm{N} / \mathrm{A}$ & $\begin{array}{l}\text { Part of a full transition } \\
\text { course led by language } \\
\text { and clinical teachers, } \\
\text { including 4-hour } \\
\text { English instruction, } \\
\text { 4-hour communication } \\
\text { workshops, 2-hour } \\
\text { clinical lab with } \\
\text { simulated patients, } \\
\text { and 32-hour clinical } \\
\text { placement. Topics: } \\
\text { social and colloquial } \\
\text { language. }\end{array}$ & 1 semester & None & None & None & $\begin{array}{l}\text { Student interviews } \\
\text { Teacher feedback }\end{array}$ & $\begin{array}{l}\text { No conclusive benefit } \\
\text { or harm, but } \\
\text { students reported } \\
\text { that the course was } \\
\text { useful and that it } \\
\text { was helpful to learn } \\
\text { about } \\
\text { communication } \\
\text { skills. }\end{array}$ \\
\hline Shah, $2010^{37}$ & Low & $\begin{array}{l}\text { ESL } \\
\text { anesthe- } \\
\text { siologist }\end{array}$ & 1 & $\begin{array}{l}\text { Weekly 1-hour } \\
\text { one-on-one accent } \\
\text { modification therapy } \\
\text { led by an SLP tailored } \\
\text { to trainee needs based } \\
\text { on diagnostic-based } \\
\text { communication } \\
\text { numeric scores. Topics: } \\
\text { consonants, vowels, } \\
\text { prosody, and } \\
\text { suprasegmentals. }\end{array}$ & 8 weeks & $\begin{array}{l}\text { Comprehensive } \\
\text { Assessment } \\
\text { of Accented- } \\
\text { ness and } \\
\text { Intelligibility }\end{array}$ & $\begin{array}{l}\text { Comprehensive } \\
\text { Assessment } \\
\text { of Accented- } \\
\text { ness and } \\
\text { Intelligibility }\end{array}$ & $\begin{array}{l}\text { Language } \\
\text { background } \\
\text { questionnaire }\end{array}$ & $\begin{array}{l}\text { Intelligibility } \\
\text { assessed by SLP } \\
\text { and } 3 \text { untrained } \\
\text { listeners } \\
\text { Pretherapy and } \\
\text { posttherapy audio } \\
\text { comparisons } \\
\text { Trainee exit } \\
\text { interview }\end{array}$ & $\begin{array}{l}\text { Improvement in } \\
\text { intelligibility, } \\
\text { intonation, stress, } \\
\text { and vowel and } \\
\text { consonant } \\
\text { production. } \\
\text { Improved comfort } \\
\text { and confidence with } \\
\text { patients and } \\
\text { presentation. } \\
\text { Program satisfaction. }\end{array}$ \\
\hline $\begin{array}{l}\text { Boughton } \\
\text { et al, } \\
2010^{40}\end{array}$ & Low & $\begin{array}{l}\text { CALD } \\
\text { nursing } \\
\text { students } \\
\text { (post- } \\
\text { gradu- } \\
\text { ate) }\end{array}$ & 17 & $\begin{array}{l}\text { Part of CALD support } \\
\text { program, led by } 3 \\
\text { nursing faculty } \\
\text { members. Five-hour } \\
\text { introductory workshop } \\
+91.5 \text {-hour sessions. } \\
\text { Topic: colloquial } \\
\text { language. }\end{array}$ & 1 semester & None & None & None & Student interviews & $\begin{array}{l}\text { Learning about } \\
\text { effective } \\
\text { communication and } \\
\text { colloquial language } \\
\text { enabled students to } \\
\text { better understand } \\
\text { various } \\
\text { colloquialisms used } \\
\text { by patients. }\end{array}$ \\
\hline
\end{tabular}


Table 3. Continued

\begin{tabular}{|c|c|c|c|c|c|c|c|c|c|c|}
\hline \multirow[b]{2}{*}{ Study } & \multirow{2}{*}{$\begin{array}{l}\text { Study } \\
\text { Quality }\end{array}$} & \multicolumn{2}{|c|}{ Participants } & \multicolumn{2}{|c|}{ Accent Intervention } & \multicolumn{2}{|c|}{ Objective Instruments } & \multicolumn{2}{|c|}{ Subjective Measures } & \multirow[b]{2}{*}{ Effect Results } \\
\hline & & Type & $\mathbf{n}$ & Content/Structure & Duration & Pretest & Posttest & Pretest & Posttest & \\
\hline $\begin{array}{l}\text { Cross and } \\
\text { Smalldridge, } \\
2011^{7}\end{array}$ & Low & IMGs & 17 & $\begin{array}{l}3 \text { sessions ( } 2 \text { half-days } \\
+1 \text { full-day) with } \\
\text { feedback by clinical } \\
\text { and language } \\
\text { teachers. Content: } \\
\text { adjustments to } \\
\text { accents and tone of } \\
\text { voice. Structure: } \\
\text { presentation, } \\
\text { workshops, practice. }\end{array}$ & $\mathrm{N} / \mathrm{A}$ & None & None & $\begin{array}{l}\text { Formal feedback } \\
\text { from } \\
\text { supervising } \\
\text { consultants }\end{array}$ & $\begin{array}{l}\text { Trainee feedback } \\
\text { Trainer feedback }\end{array}$ & $\begin{array}{l}\text { Improved } \\
\text { communication } \\
\text { skills. }\end{array}$ \\
\hline $\begin{array}{l}\text { Kokkinn and } \\
\text { Stupans, } \\
2011^{43}\end{array}$ & Low & $\begin{array}{l}\text { ESL phar- } \\
\text { macy } \\
\text { students }\end{array}$ & 82 & $\begin{array}{l}\text { Weekly 2-hour classes } \\
\text { led by clinical and } \\
\text { language specialists. } \\
\text { Content: } \\
\text { terminology, } \\
\text { colloquialism, and } \\
\text { pronunciation. }\end{array}$ & 8 weeks & None & $\begin{array}{l}\text { Final pharmacy } \\
\text { counseling test }\end{array}$ & None & $\begin{array}{l}\text { Trainee } \\
\text { self-evaluation of } \\
\text { progress } \\
\text { Trainee survey of the } \\
\text { program } \\
\text { Trainer feedback }\end{array}$ & $\begin{array}{l}\text { High counseling test } \\
\text { pass rate. }\end{array}$ \\
\hline $\begin{array}{l}\text { Woodward- } \\
\text { Kron et al, } \\
2011^{47}\end{array}$ & Low & IMGs & $\mathrm{N} / \mathrm{A}$ & $\begin{array}{l}\text { Weekly 2-hour sessions } \\
\text { with role plays, } \\
\text { simulated } \\
\text { examinations, video } \\
\text { recordings, and } \\
\text { written/verbal } \\
\text { feedback (eg, } \\
\text { pitch-trace diagram } \\
\text { for stress/intonation } \\
\text { feedback). Content: } \\
\text { vocabulary, } \\
\text { grammar, sound } \\
\text { production, prosody, } \\
\text { and discourse. }\end{array}$ & 10 weeks & None & $\begin{array}{l}\text { Linguist-graded } \\
\text { video tapes on } \\
\text { progression } \\
\text { Australian } \\
\text { Medical } \\
\text { Council Clinical } \\
\text { Examination } \\
\text { pass rate }\end{array}$ & None & Trainee focus group & $\begin{array}{l}\text { Improved } \\
\text { communication } \\
\text { skills and high } \\
\text { clinical } \\
\text { examination pass } \\
\text { rate. }\end{array}$ \\
\hline
\end{tabular}


Table 3. Continued

\begin{tabular}{|c|c|c|c|c|c|c|c|c|c|c|}
\hline \multirow[b]{2}{*}{ Study } & \multirow{2}{*}{$\begin{array}{l}\text { Study } \\
\text { Quality }\end{array}$} & \multicolumn{2}{|c|}{ Participants } & \multicolumn{2}{|c|}{ Accent Intervention } & \multicolumn{2}{|c|}{ Objective Instruments } & \multicolumn{2}{|c|}{ Subjective Measures } & \multirow[b]{2}{*}{ Effect Results } \\
\hline & & Type & $n$ & Content/Structure & Duration & Pretest & Posttest & Pretest & Posttest & \\
\hline $\begin{array}{l}\text { Koch et al, } \\
2011^{53}\end{array}$ & Low & $\begin{array}{l}\text { Nursing } \\
\text { students }\end{array}$ & 52 & $\begin{array}{l}\text { Web-based glossary } \\
\text { with hyperlinks to } \\
\text { English and } \\
\text { Mandarin (written + } \\
\text { audio) and quizzes. } \\
\text { Content: teaching } \\
\text { resources, } \\
\text { vocabulary/medical } \\
\text { terminology, and } \\
\text { pronunciation. }\end{array}$ & $\mathrm{N} / \mathrm{A}$ & $\begin{array}{l}\text { English } \\
\text { Language } \\
\text { Acculturation } \\
\text { Scale }\end{array}$ & None & None & Trainee interview & $\begin{array}{l}\text { Increased confidence } \\
\text { in communication. }\end{array}$ \\
\hline $\begin{array}{l}\text { Carr and } \\
\text { DeKemel- } \\
\text { Ichikawa, } \\
2012^{21}\end{array}$ & Low & $\begin{array}{l}\text { ESL } \\
\text { nursing } \\
\text { students }\end{array}$ & 13 & $\begin{array}{l}\text { Weekly 1-hour sessions } \\
\text { led by SLP } \\
\text { postgraduate } \\
\text { student clinicians. } \\
\text { Topics: speech } \\
\text { production and } \\
\text { prosodic features of } \\
\text { general American } \\
\text { English, } \\
\text { morphosyntax, } \\
\text { American figurative } \\
\text { and abstract } \\
\text { language, and } \\
\text { nursing terminology. }\end{array}$ & 2 semesters & $\begin{array}{l}\text { Language } \\
\text { background } \\
\text { questionnaire } \\
\text { (the Rainbow } \\
\text { Passage) } \\
\text { Brief articulation } \\
\text { test }\end{array}$ & $\begin{array}{l}\text { Psycholinguistic } \\
\text { Aspects of } \\
\text { Foreign Accent }\end{array}$ & None & $\begin{array}{l}\text { Student weekly logs } \\
\text { Student } \\
\text { questionnaire } \\
\text { Faculty feedback }\end{array}$ & $\begin{array}{l}\text { Improved speech } \\
\text { sound production } \\
\text { and prosody. } \\
\text { Trainees reported } \\
\text { the program was } \\
\text { beneficial. }\end{array}$ \\
\hline $\begin{array}{l}\text { Shen et al, } \\
2012^{38} \text { or } \\
\text { Xu et al, } \\
2010^{58}\end{array}$ & Low & $\begin{array}{l}\text { CALD } \\
\text { nurses }\end{array}$ & 51 & $\begin{array}{l}\text { Weekly 2-hour sessions, } \\
\text { taught by an SLP to } \\
\text { reduce phonologic } \\
\text { errors. Content: } \\
\text { expanded from the } \\
\text { SLP's 8-week accent } \\
\text { reduction course } \\
\text { regularly taught at a } \\
\text { university. }\end{array}$ & 10 weeks & $\begin{array}{l}\text { Compton } \\
\text { Phonological } \\
\text { Assessment of } \\
\text { Foreign Accent } \\
\text { administered } \\
\text { by SLP }\end{array}$ & $\begin{array}{l}\text { Compton } \\
\text { Phonological } \\
\text { Assessment of } \\
\text { Foreign Accent } \\
\text { administered } \\
\text { by SLP }\end{array}$ & None & None & $\begin{array}{l}\text { Significantly less } \\
\text { phonetic errors } \\
\text { and linguistic } \\
\text { errors. }\end{array}$ \\
\hline
\end{tabular}


Table 3. Continued

\begin{tabular}{|c|c|c|c|c|c|c|c|c|c|c|}
\hline \multirow[b]{2}{*}{ Study } & \multirow{2}{*}{$\begin{array}{l}\text { Study } \\
\text { Quality }\end{array}$} & \multicolumn{2}{|c|}{ Participants } & \multicolumn{2}{|c|}{ Accent Intervention } & \multicolumn{2}{|c|}{ Objective Instruments } & \multicolumn{2}{|c|}{ Subjective Measures } & \multirow[b]{2}{*}{ Effect Results } \\
\hline & & Type & $\mathbf{n}$ & Content/Structure & Duration & Pretest & Posttest & Pretest & Posttest & \\
\hline $\begin{array}{l}\text { Baker and } \\
\text { Robson, } \\
2012^{49}\end{array}$ & Low & IMGs & 14 & $\begin{array}{l}15 \text { sessions of language } \\
\text { training led by a } \\
\text { language tutor } \\
\text { (accent topics: } \\
\text { pronunciation, stress, } \\
\text { and intonation) }+6 \\
\text { sessions of } \\
\text { consultation training } \\
\text { led by experienced } \\
\text { general practitioners. }\end{array}$ & 6 months & $\begin{array}{l}\text { Tutor-graded } \\
\text { language skills } \\
\text { Tutor-graded } \\
\text { consultation } \\
\text { skills }\end{array}$ & $\begin{array}{l}\text { Tutor-graded } \\
\text { language skills } \\
\text { Tutor-graded } \\
\text { consultation } \\
\text { skills }\end{array}$ & None & $\begin{array}{l}\text { Clinical supervisor } \\
\text { questionnaire } \\
\text { Trainee focus group }\end{array}$ & $\begin{array}{l}\text { Improved language } \\
\text { and consultation } \\
\text { skills. Trainees } \\
\text { reported training } \\
\text { as helpful. }\end{array}$ \\
\hline $\begin{array}{l}\text { Blackburn, } \\
2012^{50}\end{array}$ & Low & $\begin{array}{l}\text { SLP } \\
\quad \text { students }\end{array}$ & 63 & $\begin{array}{l}\text { 1-session dialect } \\
\text { instruction. Content: } \\
\text { phonological and } \\
\text { grammatical rules of } \\
\text { African American } \\
\text { English (AAE), AAE } \\
\text { video clips, practice } \\
\text { to translate standard } \\
\text { American English } \\
\text { (SAE) into AAE. }\end{array}$ & 1 day & $\begin{array}{l}\text { 30-minute } \\
\text { closed-book } \\
\text { questionnaire } \\
\text { (knowledge } \\
\text { and attitude) }\end{array}$ & $\begin{array}{l}\text { 30-minute } \\
\text { closed-book } \\
\text { questionnaire } \\
\text { (knowledge } \\
\text { and attitude) }\end{array}$ & None & None & $\begin{array}{l}\text { Improved knowledge } \\
\text { of the AAE rules } \\
\text { (though }<50 \% \\
\text { accuracy posttest); } \\
\text { more aware of } \\
\text { difficulties children } \\
\text { face in school if } \\
\text { they don't speak } \\
\text { SAE. }\end{array}$ \\
\hline $\begin{array}{l}\text { Belay, } \\
2013^{33}\end{array}$ & Low & ESL nurses & 14 & $\begin{array}{l}\text { Weekly } 1.5 \text { - or 2-hour } \\
\text { sessions led by an } \\
\text { SLP. Content: based } \\
\text { on the Compton } \\
\text { Pronouncing English } \\
\text { as a Second } \\
\text { Language program. }\end{array}$ & 13 weeks & None & None & None & $\begin{array}{l}\text { Trainee interview } \\
(4-8 \text { months after } \\
\text { training })\end{array}$ & $\begin{array}{l}\text { Increased confidence } \\
\text { in language } \\
\text { abilities; improved } \\
\text { communication } \\
\text { experiences. }\end{array}$ \\
\hline
\end{tabular}


Table 3. Continued

\begin{tabular}{|c|c|c|c|c|c|c|c|c|c|c|}
\hline \multirow[b]{2}{*}{ Study } & \multirow{2}{*}{$\begin{array}{l}\text { Study } \\
\text { Quality }\end{array}$} & \multicolumn{2}{|c|}{ Participants } & \multicolumn{2}{|c|}{ Accent Intervention } & \multicolumn{2}{|c|}{ Objective Instruments } & \multicolumn{2}{|c|}{ Subjective Measures } & \multirow[b]{2}{*}{ Effect Results } \\
\hline & & Type & $\mathbf{n}$ & Content/Structure & Duration & Pretest & Posttest & Pretest & Posttest & \\
\hline $\begin{array}{c}\text { Khurana and } \\
\text { Huang, } \\
2013^{36}\end{array}$ & Low & $\begin{array}{l}\text { IMGs and } \\
\text { IMRs }\end{array}$ & 82 & $\begin{array}{l}\text { Weekly } 90 \text { - to } \\
\text { 120-minute classes } \\
\text { (size: 8-14) led by } \\
\text { accent-reduction } \\
\text { instructors. Topics: } \\
\text { vowels and } \\
\text { consonants, fluency, } \\
\text { stress, rhythm, } \\
\text { intonation and } \\
\text { casual speech } \\
\text { linking, blending, } \\
\text { contractions, and } \\
\text { reductions. }\end{array}$ & $\begin{array}{l}8 \text { to } 12 \\
\text { weeks }\end{array}$ & $\begin{array}{l}\text { Video/audio tape } \\
\text { reviewed by } 2 \\
\text { external } \\
\text { assessors }\end{array}$ & $\begin{array}{l}\text { Video/audio tape } \\
\text { reviewed by } 2 \\
\text { external } \\
\text { assessors }\end{array}$ & $\begin{array}{l}\text { Trainee } \\
\text { self-evaluation } \\
\text { of communi- } \\
\text { cation } \\
\text { skills }\end{array}$ & $\begin{array}{l}\text { Trainee } \\
\text { self-evaluation of } \\
\text { communication } \\
\text { skills }\end{array}$ & $\begin{array}{l}\text { Significantly } \\
\text { improved ability to } \\
\text { pronounce words } \\
\text { distinctly and } \\
\text { stress more } \\
\text { accurately; better } \\
\text { accuracy, } \\
\text { intonation, fluency, } \\
\text { speed, and volume. } \\
\text { Increased } \\
\text { confidence in } \\
\text { communication. }\end{array}$ \\
\hline $\begin{array}{c}\text { Harvey et al, } \\
2013^{42}\end{array}$ & Low & $\begin{array}{l}\text { CALD } \\
\text { nursing } \\
\text { students } \\
\text { (post- } \\
\text { gradu- } \\
\text { ate) }\end{array}$ & 240 & $\begin{array}{l}6 \text { weeks of weekly } \\
\text { 2-hour interactive } \\
\text { discussion sessions, } \\
+4 \text { weeks of role } \\
\text { plays led by } \\
\text { language and clinical } \\
\text { facilitators ( } 18 \text { hours } \\
\text { total). Topics: } \\
\text { listening, speaking, } \\
\text { and vocabulary. }\end{array}$ & 10 weeks & None & $\begin{array}{l}\text { Clinical practice } \\
\text { pass rate }\end{array}$ & None & $\begin{array}{l}\text { Trainee survey after } \\
\text { 6-week discussion } \\
\text { sessions } \\
\text { Trainee survey after } \\
10 \text { weeks } \\
\text { Trainee survey } 1 \\
\text { week after clinical } \\
\text { placement } \\
\text { completion }\end{array}$ & $\begin{array}{l}\text { Similar to or higher } \\
\text { than overall cohort } \\
\text { pass rate for clinical } \\
\text { practice. Increased } \\
\text { confidence and } \\
\text { comfort in } \\
\text { communication } \\
\text { and enhanced } \\
\text { comprehension of } \\
\text { Australian } \\
\text { colloquial } \\
\text { language. }\end{array}$ \\
\hline
\end{tabular}


Table 3. Continued

\begin{tabular}{|c|c|c|c|c|c|c|c|c|c|c|}
\hline \multirow[b]{2}{*}{ Study } & \multirow{2}{*}{$\begin{array}{l}\text { Study } \\
\text { Quality }\end{array}$} & \multicolumn{2}{|c|}{ Participants } & \multicolumn{2}{|c|}{ Accent Intervention } & \multicolumn{2}{|c|}{ Objective Instruments } & \multicolumn{2}{|c|}{ Subjective Measures } & \multirow[b]{2}{*}{ Effect Results } \\
\hline & & Type & $\mathbf{n}$ & Content/Structure & Duration & Pretest & Posttest & Pretest & Posttest & \\
\hline $\begin{array}{l}\text { Van Schaik } \\
\text { et al, } \\
2014^{54}\end{array}$ & Low & $\begin{array}{l}\text { CALD } \\
\text { nursing } \\
\text { students } \\
\text { and } \\
\text { CALD } \\
\text { nurses }\end{array}$ & 19 & $\begin{array}{l}\text { 8-hour practice in } \\
\text { online course (3 } \\
\text { 20-minute practices } \\
\text { per week). Structure: } \\
\text { instructions, } \\
\text { exercises, tips, and } \\
\text { feedback. Topics: } \\
\text { phonological and } \\
\text { prosodic rules that } \\
\text { govern American } \\
\text { English } \\
\text { pronunciation and } \\
\text { intonation, vowels, } \\
\text { consonants, } \\
\text { common idioms, and } \\
\text { figures of speech } \\
\text { relevant in } \\
\text { healthcare. }\end{array}$ & 3 months & $\begin{array}{l}\text { Online Test of } \\
\text { English } \\
\text { Proficiency } \\
\text { Level } \\
\text { Knowledge test } \\
\text { Proficiency in } \\
\text { Oral English } \\
\text { Communica- } \\
\text { tion - } \\
\text { Screen }\end{array}$ & $\begin{array}{l}\text { Knowledge test } \\
\text { Proficiency in } \\
\text { Oral English } \\
\text { Communica- } \\
\text { tion - } \\
\text { Screen }\end{array}$ & None & $\begin{array}{l}\text { Trainee survey } \\
\text { Trainee exit } \\
\text { interview }\end{array}$ & $\begin{array}{l}\text { Significant gains in } \\
\text { knowledge and } \\
\text { verbal } \\
\text { performance; } \\
\text { program } \\
\text { satisfaction. }\end{array}$ \\
\hline $\begin{array}{l}\text { Freysteinson } \\
\text { et al, } \\
2017^{34}\end{array}$ & Low & $\begin{array}{l}\text { CALD } \\
\text { health } \\
\text { students } \\
\text { and } \\
\text { CALD } \\
\text { nurses }\end{array}$ & 27 & $\begin{array}{l}\text { Weekly 1-hour sessions } \\
\text { taught by an SLP. } \\
\text { Topics: role of } \\
\text { prosody in } \\
\text { acquisition of } \\
\text { American English } \\
\text { pronunciation, vowel } \\
\text { and consonant } \\
\text { sounds, and North } \\
\text { American English } \\
\text { medical terminology. }\end{array}$ & 12 weeks & $\begin{array}{l}\text { Self-Esteem Scale } \\
\text { Personal Report } \\
\text { of Communi- } \\
\text { cation } \\
\text { Apprehension- } \\
24 \\
\text { Self-Perceived } \\
\text { Communica- } \\
\text { tive } \\
\text { Competence }\end{array}$ & $\begin{array}{l}\text { Self-Esteem Scale } \\
\text { Personal Report } \\
\text { of Communi- } \\
\text { cation } \\
\text { Apprehension- } \\
24 \\
\text { Self-Perceived } \\
\text { Communica- } \\
\text { tive } \\
\text { Competence }\end{array}$ & None & None & $\begin{array}{l}\text { Significantly higher } \\
\text { self-esteem and } \\
\text { overall } \\
\text { competence } \\
\text { related to } \\
\text { communication. }\end{array}$ \\
\hline
\end{tabular}


students ( $n=737,76 \%$ ) majoring in nursing, medicine, pharmacy, speech-language pathology, or other health sciences. The second-largest group consisted of 143 (15\%) prelicensed health professionals, such as international medical graduates, international nursing graduates, and international medical researchers. In addition, 84 participants (9\%) were practicing health professionals, including licensed clinicians, administrators, and clerks.

\section{Intervention Effects}

Beneficial Effects. All but 2 studies demonstrated some specific benefits from accent interventions; however, the 2 inconclusive studies reported student trainees' satisfaction with the intervention. ${ }^{41,52}$ Identified benefits included improvements in speech production and communication competence, improvements in academic or clinical performance, and positive perceptual changes, as summarized in Table 4 . The majority of the studies $(n=20,77 \%)$ indicated program satisfaction based on trainee or trainer feedback.

Adverse Effects. Adverse effects or unintended consequences were seldom discussed in the included studies. No objective measures of adverse events were reported, but 3 studies provided qualitative comments on the topic. In a study with culturally and linguistically diverse nurses, the length of the accent reduction training program (10 weeks) was reported to have fatigued some participants; the authors also observed that videotaping encounters with standardized patients added anxiety for some participants. ${ }^{58}$ In a language-training program for international medical graduates, disruption of normal work patterns in small departments was mentioned. ${ }^{49}$ The third study reporting adverse effects implemented a web-based training program for nursing students, and the author reported "two students felt marginalized and suggested that more emphasis be placed on creating opportunities for them to communicate with local students." 53

Medium-Term Effects. Most studies reported only shortterm effect data collected immediately or almost immediately after completion of the intervention. The longest follow-up period was 2 semesters after the completion of a 2-semester supplementary support program for nursing students. $^{22}$ In another study, trainees were interviewed 4 to 8 months after a 13-week training. ${ }^{33}$ In another study, a trainee questionnaire survey was administered 3 months after a 2semester training. ${ }^{46}$ These 3 studies all reported positive trainee feedback on the intervention effects.

\section{Quality of the Included Studies}

As shown in Table 3, all included studies were of low research quality and often had small sample sizes and few objective outcome measures, indicating a lack of generalizability and reproducibility. Only one study-a controlled before-after study-had a control group. ${ }^{38}$ Most studies ( $n=22,85 \%)$ were case series (also known as uncontrolled longitudinal studies), which were before-after studies with no comparison group as a control. The remaining 3 studies $^{35,37,55}$ had a case study design.

A range of objective and subjective outcome measures was used in the included studies. Notably, in every one of the 26 studies, a different set of evaluation measures was chosen or developed. Eighteen studies (69\%) tested some objective measure (pretest and/or posttest); 8 (31\%) reported only qualitative data. Objective posttest outcome measures used to assess intervention effects included study-specific indicators such as knowledge tests based on the content of the training program ${ }^{50,54}$ and standardized/ established instruments such as the following:

- Australian Medical Council Clinical Examination:59 Strengths include being a practical clinical test and representative of the performance and knowledge expected in clinical situations.

- Comprehensive Assessment of Accentedness and Intelligibility: ${ }^{57}$ Tool for diagnosis and evaluation of a wide variety of speech, auditory, and nonverbal features related to accents/dialects that improves upon the Compton Phonological Assessment of Foreign Accent, ${ }^{60}$ the Psycholinguistic Aspects of Foreign Accent, ${ }^{61}$ and the Proficiency in Oral English Communication - Screen ${ }^{62}$ tools by addressing wider variables and supporting pretraining diagnostics.

- Compton Phonological Assessment of Foreign Accent60 and Psycholinguistic Aspects of Foreign Accent: ${ }^{61}$ Similar tools that measure speech features relevant to accents, with a focus on phonology.

- Proficiency in Oral English Communication - Screen: ${ }^{62}$ Measures accent-related speech features and improves upon the Compton Phonological Assessment of Foreign Accent and Psycholinguistic Aspects of Foreign Accent by addressing wider speech variables such as prosody.

- Personal Report of Communication Apprehension-24:63 Focuses on self-perceived comfort/apprehension with communication.

- Self-Esteem Scale: ${ }^{64}$ Validated instrument used to measure self-esteem.

- Self-Perceived Communicative Competence: ${ }^{65}$ Focuses on self-perceived communication competence.

\section{Role of Language or Communication Experts}

Most studies included in this review involved language/ communication experts-an SLP or English teacher-in the delivery of accent interventions. These experts led the training either as a sole instructor $21,33,34,36-38,45,49,50$ or as part of a multidisciplinary teaching team along with a clinical teacher. ${ }^{7,22,41-44,47,48}$ The latter collaborative teaching style was unanimously praised and recommended in all of the studies that used this style. ESL teachers, with understanding of linguistic complexity, are able to teach aspects of language in detail with a specific curriculum, goals, and lesson plans. SLPs, with expertise and experience in speech and language therapy, are also ideally suited to conduct regional or foreign accent improvement programs. ${ }^{66}$

\section{DISCUSSION \\ Effects and Need for Accent Interventions}

Despite the lack of standardized outcome measures in the studies included in this review, the current evidence suggests that accent interventions in the healthcare context can have direct benefits on communication quality: improvement in speech production, clarity, intelligibility, listening comprehension, knowledge of colloquialism and phonological rules, and reduction of phonological errors. Indirect benefits include improved academic and clinical performance and positive perceptual changes, such as 
Table 4. Beneficial Effects of Accent Interventions

\begin{tabular}{|c|c|c|}
\hline Key Themes & Beneficial Effects & Studies \\
\hline \multirow[t]{3}{*}{ Speech production } & $\begin{array}{l}\text { Better communication clarity } \\
\text { and/or improved } \\
\text { communication experiences }\end{array}$ & $\begin{array}{l}\text { Bosher and Smalkoski, } 2002^{39} \\
\text { Satter et al, } 2005^{55} \\
\text { San Miguel et al, } 2006^{45} \\
\text { Parkhurst, } 2007^{44} \\
\text { Seibold et al, } 2007^{46} \\
\text { Shah, } 2010^{37} \\
\text { Boughton et al, 2010 } \\
\text { Cross and Smalldridge, } 2011^{7} \\
\text { Woodward-Kron et al, } 2011^{47} \\
\text { Carr and DeKemel-Ichikawa, } 2012^{21} \\
\text { Baker and Robson, } 2012^{49} \\
\text { Belay, 2013 } \\
\text { Harvey et al, 2013 } \\
\text { Freysteinson et al, } 2017^{34}\end{array}$ \\
\hline & Increased intelligibility & $\begin{array}{l}\text { Parkhurst, } 2007^{44} \\
\text { Shah, } 2010^{37} \\
\text { Woodward-Kron et al, } 2011^{47} \\
\text { Carr and DeKemel-Ichikawa, } 2012^{21} \\
\text { Baker and Robson, } 2012^{49} \\
\text { Khurana and Huang, 2013 } \\
\text { Van Schaik et al, } 2014^{54}\end{array}$ \\
\hline & Fewer phonological errors & $\begin{array}{l}\text { Shah, } 2010^{37} \\
\text { Carr and DeKemel-Ichikawa, } 2012^{21} \\
\text { Shen et al, } 2012^{38} \\
\text { Baker and Robson, } 2012^{49} \\
\text { Khurana and Huang, } 2013^{36} \\
\text { Van Schaik et al, 2014 }\end{array}$ \\
\hline \multirow[t]{3}{*}{ Communication competence } & $\begin{array}{l}\text { Better listening comprehension } \\
\text { ability }\end{array}$ & $\begin{array}{l}\text { Guhde, } 2003^{35} \\
\text { Parkhurst, } 2007^{44} \\
\text { Shah, } 2010^{37} \\
\text { Baker and Robson, } 2012^{49} \\
\text { Harvey et al, } 2013^{42}\end{array}$ \\
\hline & $\begin{array}{l}\text { Improved knowledge of } \\
\text { colloquial language }\end{array}$ & $\begin{array}{l}\text { Chur-Hansen and Barrett, } 1996^{51} \\
\text { Shah, } 2010^{37} \\
\text { Boughton et al, } 2010^{40} \\
\text { Van Schaik et al, } 2014^{54}\end{array}$ \\
\hline & $\begin{array}{l}\text { Improved knowledge of } \\
\text { phonological rules }\end{array}$ & $\begin{array}{l}\text { Shah, } 2010^{37} \\
\text { Blackburn, } 2012^{50} \\
\text { Van Schaik et al, } 2014^{54}\end{array}$ \\
\hline \multirow[t]{4}{*}{ Academic/clinical performance } & $\begin{array}{l}\text { Improved academic/clinical exam } \\
\text { pass rate }\end{array}$ & $\begin{array}{l}\text { Symes et al, } 2002^{22} \\
\text { Bosher and Smalkoski, } 2002^{39} \\
\text { San Miguel et al, } 2006^{45} \\
\text { Parkhurst, } 2007^{44} \\
\text { Kokkinn and Stupans, } 2011^{43} \\
\text { Woodward-Kron et al, } 2011^{47} \\
\text { Harvey et al, } 2013^{42}\end{array}$ \\
\hline & Higher student retention rate & Symes et al, $2002^{22}$ \\
\hline & Less clinical incidents & Yahes and Dunn, $1996^{48}$ \\
\hline & Better health survey response rate & Satter et al, $2005^{55}$ \\
\hline Perceptual changes & $\begin{array}{l}\text { Increased confidence and/or } \\
\text { self-esteem in communication }\end{array}$ & $\begin{array}{l}\text { Symes et al, } 2002^{22} \\
\text { San Miguel et al, } 2006^{45} \\
\text { Boughton et al, } 2010^{40} \\
\text { Koch et al, } 2011^{53} \\
\text { Belay, } 2013^{33} \\
\text { Khurana and Huang, } 2013^{36}\end{array}$ \\
\hline
\end{tabular}




\begin{tabular}{|c|c|c|}
\hline Key Themes & Beneficial Effects & Studies \\
\hline & & $\begin{array}{l}\text { Harvey et al, } 2013^{42} \\
\text { Freysteinson et al, } 2017^{34}\end{array}$ \\
\hline & Improved collegiality & $\begin{array}{l}\text { Yahes and Dunn, } 1996^{48} \\
\text { Guhde, } 2003^{35} \\
\text { Boughton et al, } 2010^{40} \\
\text { Carr and DeKemel-Ichikawa, } 2012^{21} \\
\text { Harvey et al, } 2013^{42}\end{array}$ \\
\hline & $\begin{array}{l}\text { Improved attitude towards } \\
\text { people with accent/dialect }\end{array}$ & $\begin{array}{l}\text { Satter et al, } 2005^{55} \\
\text { Blackburn, } 2012^{50}\end{array}$ \\
\hline & Increased job satisfaction & Yahes and Dunn, $1996^{48}$ \\
\hline
\end{tabular}

improved collegiality and job satisfaction. The demonstrated range of beneficial effects provides a strong case for implementing accent-related communication improvement strategies in healthcare to support the diverse healthcare workforce serving increasingly diverse populations.

Long-term effects and adverse effects were seldom measured in the included studies. Only 3 studies reported qualitative comments that identified problems with participant fatigue and anxiety, ${ }^{58}$ disruption of normal clinical work, ${ }^{49}$ and feeling marginalized. ${ }^{53}$ We recommend that future studies further explore such issues and comprehensively assess the intervention impact on the participants. Documenting such unintended consequences and long-term effects is important because accent interventions are largely unregulated and free of the jurisdiction of any particular clinical practice area. In other words, no specialized license or accreditation requirements and no guiding ethical code of conduct apply to this area of practice. Accent modification services, for instance, are offered by a variety of practitioners, including licensed professionals (SLPs), ESL teachers, and nonprofessionals. Overidentification and underidentification of participants are possible in accent modification referrals. Exclusionary and discriminatory marginalization of a culturally and linguistically diverse individual, simply on the basis of an accent that differs from the local norm, is a problem of linguistic profiling. ${ }^{67}$ We recommend that future studies of accent interventions document the participant selection criteria and plan for rigorous assessment of both beneficial and adverse effects in the short and long term.

\section{Lack of Standardized Outcome Measurements}

Every included study used a different set of evaluation measures, ranging from objective measures of speech production, to clinical examinations with standardized patients, to qualitative feedback. The lack of control groups and the lack of comparison from pretest to posttest using consistent objective instruments limit the strength of the current evidence base. The substantial heterogeneity in effect measures reflects a lack of standardized assessment tools in this field and a lack of methodological rigor to support generalizability and reproducibility.

The objective assessment tools that were used in some of the studies have strengths and weaknesses that require a careful examination for adoption in accent interventions. Future research that evaluates accent interventions should consider using standardized measurement tools to pro- vide comparable findings. Moreover, none of the included studies reported findings on patient outcomes or the costeffectiveness of the accent interventions. Future research may explore these important topics to guide practice. We recommend a comprehensive assessment of accent intervention effects to measure not only the effects on communication and intelligibility improvement but also flow-on effects, including patient satisfaction and health outcomes.

\section{Variability of Accent Intervention Elements}

The accent interventions included in this review were all training programs for healthcare professionals or students, most of whom had a culturally and linguistically diverse/ESL background. The heterogeneity of the intervention design is evident in the great variety of program elements in terms of training content, structure, and duration. The linguistic topics in the training content varied from program to program, ranging from vowel and consonant production and stress and prosody training to higher-order language elements such as grammar, colloquialisms, idioms, small talk, formulaic expressions, paralinguistic features, medical terminology, and listening comprehension.

A needs assessment, often via trainee survey or focus group session, was conducted in a few studies before the accent intervention was implemented. Only one intervention, a case study of a one-on-one training program, ${ }^{37}$ was tailored to address specific speech areas based on structured pretraining diagnostics using the Comprehensive Assessment of Accentedness and Intelligibility. ${ }^{57}$ A few other studies recorded pretest speech performance based on tutor-graded consultation skills or established instruments such as the Compton Phonological Assessment of Foreign Accent ${ }^{60}$ and Proficiency in Oral English Communication - Screen. ${ }^{62}$ Future research may explore the feasibility and impact of using such instruments with medium or large groups of trainees to guide the customization of accent training programs to meet individual trainees' needs.

The inconsistency of the content and procedure among accent interventions highlights a need to develop evidencebased, best-practice guidelines in this field. Laboratory research in speech sciences suggests training in vowel production, ${ }^{68-70}$ consonant production, ${ }^{71,72}$ suprasegmental features, ${ }^{73-75}$ auditory discrimination, ${ }^{76}$ and listening comprehension. ${ }^{23,24,77}$ Rigorous evaluation research in the healthcare setting to assess the impact of accent interventions that is based on these laboratory findings may 
strengthen the evidence base and contribute to the standardization of practice.

None of the included studies reported on the scalability of their accent interventions, but the 2 fully automated web-based training programs ${ }^{53,54}$ appeared promising, with both indicating benefits according to trainee feedback. No other technology-based interventions that particularly address the accent/dialect problem in healthcare were retrieved in the search. However, general communication assistance tools are effective in this setting. For example, a paper-based visual communication tool to assist clinician-patient interaction during counseling was found effective. ${ }^{78}$ Giving patients a printout of their results or a written explanation to assist during encounters with accented physicians has been suggested. ${ }^{14}$ Such visual assistive tools (paper-based or technology-based) might be useful in addressing communication barriers related to linguistic diversity and might be worth testing further. Future research should also gather evidence on the cost-effectiveness of accent interventions whether technology-based or not.

\section{Implication for Accent Intervention Practice}

Accent interventions in healthcare should aim for improvement in speech production in terms of clarity and intelligibility, listening comprehension ability, knowledge of colloquialism and phonological rules, and reduction of phonological errors. Further goals of accent intervention may include better academic or clinical performance and positive perceptual change.

Accent training, ideally delivered by an SLP, may plan for curriculum content on vowel and consonant production, suprasegmental features, phonological variation, auditory discrimination, listening comprehension, vocabulary variability, nonverbal cues, and pragmatic cues (ie, social use of language). Decisions regarding other accent intervention elements may also require careful consideration in terms of the target population, intervention structure, duration, and delivery media, as well as diagnostics and assessment of trainee performance, ideally using objective tools. The rationale for each decision should be documented and reviewed posttraining to assist with the intervention evaluation. The evaluation should also identify the program impact on patient outcomes and health system outcomes, including adverse or unintended consequences in the short and long terms.

\section{Study Limitations}

The topic of this review was limited to spoken language diversity in the healthcare setting, especially related to accent and dialect. We did not consider other aspects of communication differences, such as nonverbal linguistic diversity and other dimensions of cultural competence in healthcare. We also excluded translation, interpretation, and transcribing services provided by humans or technology. By including only the interventions that had an element to address accent- or dialect-related communication problems, we aimed to add specific knowledge regarding the efficacy of accent-targeted interventions in healthcare.

The clear heterogeneity of the accent interventions and outcome measures prevented any meaningful metaanalysis of the current data. No randomized controlled trials on accent intervention in the health setting were identified. Instead of reporting risk of bias in each study, we enhanced the review rigor via robust study inclusion criteria and study quality assessment criteria based on the literature and clinical expertise. All included studies had low quality, highlighting the need to strengthen the evidence base in the accent intervention evaluation field. We recommend implementing large randomized controlled studies that not only measure the intervention effects with standardized instruments but also collect data on cost-effectiveness.

\section{CONCLUSION}

Despite the low research quality and lack of standardized measures in the included studies, this review establishes a sufficient evidence base to advocate for implementing accent-related communication improvement strategies in healthcare. The key benefits associated with accent interventions include improvements in speech production, communication competence, and academic/clinical performance, as well as perceptual changes. Large randomized controlled studies would be helpful to strengthen the evidence base regarding accent interventions in healthcare.

\section{ACKNOWLEDGMENTS}

The authors have no financial or proprietary interest in the subject matter of this article.

\section{REFERENCES}

1. Ha JF, Longnecker N. Doctor-patient communication: a review. Ochsner J. 2010 Spring;10(1):38-43.

2. Divi C, Koss RG, Schmaltz SP, Loeb JM. Language proficiency and adverse events in US hospitals: a pilot study. Int J Qual Health Care. 2007 Apr;19(2):60-67. doi: 10.1093/intqhc/mzl069.

3. Stewart MA. Effective physician-patient communication and health outcomes: a review. CMAJ. 1995 May 1;152(9):1423-1433.

4. O'Daniel M, Rosenstein AH. Professional Communication and Team Collaboration. In: Hughes RG, ed. Patient Safety and Quality: An Evidence-Based Handbook for Nurses. Rockville, MD: Agency for Healthcare Research and Quality (US); 2008.

5. Amon E. Communication strategies for reducing hospital error and professional liability. Obstet Gynecol Surv. 2002 Nov;57(11):713-714.

6. Aiken LH. U.S. nurse labor market dynamics are key to global nurse sufficiency. Health Serv Res. 2007 Jun;42(3 Pt 2):1299-1320. doi: 10.1111/j.1475-6773.2007.00714.x.

7. Cross $D$, Smalldridge A. Improving written and verbal communication skills for international medical graduates: a linguistic and medical approach. Med Teach. 2011;33(7):e364-e367. doi: 10.3109/0142159X.2011.577469.

8. Austrailian Government Department of Health. Report on the audit of health workforce in rural and regional Australia. www.health.gov.au/internet/publications/publishing.nsf/ Content/work-res-ruraud-toc $\sim$ work-res-ruraud-1 $\sim$ work-resruraud-1-7. Updated April 2008. Accessed January 30, 2017.

9. Institute of Medicine Committee on the Adequacy of Nursing Staff in Hospitals and Nursing Homes. Implications of Population Change. In: Wunderlich GS, Sloan F, Davis CK, eds. Nursing Staff in Hospitals and Nursing Homes: Is It Adequate? Washington, DC: National Academies Press; 1996.

10. Dias S, Gama A, Cargaleiro H, Martins MO. Health workers' attitudes toward immigrant patients: a cross-sectional survey 
in primary health care services. Hum Resour Health. $2012 \mathrm{Jul}$ 9;10:14. doi: 10.1186/1478-4491-10-14.

11. ASHA Joint Subcommittee of the Executive Board on English Language Proficiency. Students and professionals who speak English with accents and nonstandard dialects: issues and recommendations [Technical Report]. American Speech-Language-Hearing Association. www.asha.org/policy/TR1998-00154. Accessed October 6, 2017.

12. Wolfram W, Fasold RW. The Study of Social Dialects in American English. Englewood Cliffs, NJ: Prentice-Hall; 1974.

13. Wagner LM, Brush BL, Castle NG, Eaton M, Capezuti E. Examining differences in nurses' language, accent, and comprehensibility in nursing home settings based on birth origin and country of education. Geriatr Nurs. 2015 Jan-Feb;36(1):47-51. doi: 10.1016/j.gerinurse.2014.10.012.

14. Sommer J, Macdonald W, Bulsara C, Lim D. Grunt language versus accent: the perceived communication barriers between international medical graduates and patients in Central Wheatbelt catchments. Aust J Prim Health. 2012;18(3):197-203. doi: 10.1071/PY11030.

15. Valles B. The Impact of Accented English on Speech Comprehension. El Paso, TX: ETD Collection for University of Texas, University of Texas; 2015.

16. Horani LA. The Effect of a Physician's Pronunciation on Nurses' Perceptions of the Physician's Medical Competency. Portland, OR: Portland State University; 1995.

17. Butow $P$, Bell $M$, Goldstein D, et al. Grappling with cultural differences; communication between oncologists and immigrant cancer patients with and without interpreters. Patient Educ Couns. 2011 Sep;84(3):398-405. doi: 10.1016/j.pec.2011.01.035.

18. Mangrio E, Sjögren Forss K. Refugees' experiences of healthcare in the host country: a scoping review. BMC Health Serv Res. 2017 Dec 8;17(1):814. doi: 10.1186/s12913-017-2731-0.

19. Fuertes JN, Gelso CJ. Hispanic counselors' race and accent and Euro Americans' universal-diverse orientation: a study of initial perceptions. Cultur Divers Ethnic Minor Psychol. 2000 May;6(2):211-219.

20. Kramer M. Educational challenges of international medical graduates in psychiatric residencies. J Am Acad Psychoanal Dyn Psychiatry. 2006 Spring;34(1):163-171. doi: 10.1521/jaap.2006.34.1.163.

21. Carr SM, DeKemel-Ichikawa K. Improving communication through accent modification: growing the nursing workforce. J Cult Divers. 2012 Fall;19(3):79-84.

22. Symes L, Tart K, Travis L, Toombs MS. Developing and retaining expert learners: the student success program. Nurse Educ. 2002 Sep-Oct;27(5):227-231.

23. Baese-Berk MM, Bradlow AR, Wright BA. Accent-independent adaptation to foreign accented speech. J Acoust Soc Am. 2013 Mar;133(3):EL174-80. doi: 10.1121/1.4789864.

24. Adank $P$, Hagoort $P$, Bekkering $H$. Imitation improves language comprehension. Psychol Sci. 2010;21(12):1903-1909.

25. Chan A, Purcell A, Power E. A systematic review of assessment and intervention strategies for effective clinical communication in culturally and linguistically diverse students. Med Educ. 2016 Sep;50(9):898-911. doi: 10.1111/medu.13117.

26. Higgins JPT, Green S, eds. Cochrane Handbook for Systematic Reviews of Interventions, ver 5.1.0. The Cochrane Collaboration, 2011. www.handbook.cochrane.org. Updated March 2011. Accessed January 24, 2017.

27. Crowther M, Lim W, Crowther MA. Systematic review and meta-analysis methodology. Blood. 2010 Oct 28;116(17):3140-3146. doi: 10.1182/blood-2010-05-280883.
28. Thomas J, Harden A, Oakley A, et al. Integrating qualitative research with trials in systematic reviews. BMJ. $2004 \mathrm{Apr}$ 24;328(7446):1010-1012. doi: 10.1136/bmj.328.7446.1010.

29. Moher D, Liberati A, Tetzlaff J, Altman DG; PRISM Group. Preferred reporting items for systematic reviews and meta-analyses: the PRISMA statement. PLoS Med. 2009 Jul 21;6(7):e1000097. doi: 10.1371/journal.pmed.1000097.

30. Burns PB, Rohrich RJ, Chung KC. The levels of evidence and their role in evidence-based medicine. Plast Reconstr Surg. 2011 Jul;128(1):305-310. doi: 10.1097/PRS.0b013e318219c171.

31. Atkins D, Best D, Briss PA, et al. GRADE Working Group. Grading quality of evidence and strength of recommendations. BMJ. 2004 Jun 19;328(7454):1490.

32. Cochrane Effective Practice and Organisation of Care (EPOC). Suggested risk of bias criteria for EPOC reviews. epoc.cochrane.org/sites/epoc.cochrane.org/files/public/ uploads/Resources-for-authors2017/suggested _risk_of_bias_criteria_for_epoc_reviews.pdf. Published August 22, 2017. Accessed June 7, 2019.

33. Belay HA. A Qualitative Investigation of the Communication Experiences of International Nurses Who Participated in a 13-Week Accent Modification Program. Denton, TX: College of Nursing, Texas Woman's University; 2013.

34. Freysteinson WM, Adams JD, Cesario S, et al. An accent modification program. J Prof Nurs. 2017 Jul-Aug;33(4):299-304. doi: 10.1016/j.profnurs.2016.11.003.

35. Guhde JA. English-as-a-second language (ESL) nursing students: strategies for building verbal and written language skills. J Cult Divers. 2003 Winter;10(4):113-117.

36. Khurana $P$, Huang E. Efficacy of accent modification training for international medical professionals. J Univ Teach Learning Pract. 2013 Aug 30;10(2).

37. Shah AP. An International Medical Doctor in Practice: A Representative Case Study of a High English Proficiency Client for Accent Modification. In: Chabon S, Cohn E, eds. Communication Disorders: A Case-Based Approach: Stories From the Front Line. New York, NY: Allyn \& Bacon; 2010:393-402.

38. Shen JJ, Xu Y, Bolstad AL, Covelli M, Torpey M, Colosimo R. Effects of a short-term linguistic class on communication competence of international nurses: implications for practice, policy, and research. Nurs Econ. 2012 Jan-Feb;30(1):21-28.

39. Bosher S, Smalkoski K. From needs analysis to curriculum development: designing a course in health-care communication for immigrant students in the USA. Engl Specif Purp. 2002;21(1):59-79. doi: 10.1016/S0889-4906(01)00002-3.

40. Boughton MA, Halliday LE, Brown L. A tailored program of support for culturally and linguistically diverse (CALD) nursing students in a graduate entry masters of nursing course: a qualitative evaluation of outcomes. Nurse Educ Pract. 2010 Nov;10(6):355-360. doi: 10.1016/j.nepr.2010.05.003.

41. Chiang V, Crickmore BL. Improving English proficiency of post-graduate international nursing students seeking further qualifications and continuing education in foreign countries. J Contin Educ Nurs. 2009 Jul;40(7):329-336.

42. Harvey T, Robinson C, Frohman R. Preparing culturally and linguistically diverse nursing students for clinical practice in the health care setting. J Nurs Educ. 2013 Jul;52(7):365-370. doi: 10.3928/01484834-20130529-02.

43. Kokkinn B, Stupans I. Improving pharmacy counselling skills: an interdisciplinary model of support for students with English as an additional language. Int J Pharm Pract. 2011 Dec;19(6):435-437. doi: 10.1111/j.2042-7174.2011.00149.x.

44. Parkhurst $C$. A communication course for a linguistically diverse student population. Am J Pharm Educ. $2007 \mathrm{Apr}$ 15;71(2):36. doi: 10.5688/aj710236. 
45. San Miguel C, Rogan F, Kilstoff K, Brown D. Clinically speaking: a communication skills program for students from non-English speaking backgrounds. Nurse Educ Pract. 2006 Sep;6(5):268-274. doi: 10.1016/j.nepr.2006.02.004.

46. Seibold C, Rolls C, Campbell M. Nurses on the move: evaluation of a program to assist international students undertaking an accelerated bachelor of nursing program. Contemp Nurse. 2007 May-Jun;25(1-2):63-71.

47. Woodward-Kron R, Stevens M, Flynn E. The medical educator, the discourse analyst, and the phonetician: a collaborative feedback methodology for clinical communication. Acad Med. 2011 May;86(5):565-570. doi: 10.1097/ACM.0b013e318212feaf.

48. Yahes E, Dunn AK. Enculturation of foreign nurse graduates: an integrated model. J Contin Educ Nurs. 1996 May-Jun;27(3):120-123.

49. Baker D, Robson J. Communication training for international graduates. Clin Teach. 2012 Oct;9(5):325-329. doi: $10.1111 /$ j.1743-498X.2012.00555.x.

50. Blackburn JF. The effect of dialect instruction on student knowledge of and attitudes toward African American English. Comm Disord Q. 2012 Aug;33(4):220-229.

51. Chur-Hansen A, Barrett RJ. Teaching colloquial Australian English to medical students from non-English speaking backgrounds. Med Educ. 1996 Nov;30(6):412-417.

52. Chur-Hansen A. Teaching support in the behavioural sciences for non-english speaking background medical undergraduates. Med Educ. 1999 Jun;33(6):404-410.

53. Koch J, Salamonson Y, Du HY, et al. Value of web-based learning activities for nursing students who speak English as a second language. J Nurs Educ. 2011 Jul;50(7):373-380. doi: 10.3928/01484834-20110331-02.

54. Van Schaik E, Lynch EM, Stoner SA, Sikorski LD. Can a web-based course improve communicative competence of foreign-born nurses? Lang Learn Technol. 2014 Feb;18(1): 11-22.

55. Satter DE, Veiga-Ermert A, Burhansstipanov L, Pena L, Restivo T. Communicating respectfully with American Indian and Alaska Natives: lessons from the California Health Interview Survey. J Cancer Educ. 2005 Spring;20(1):49-51.

56. Compton AJ. Compton P-ESL Program. San Francisco, CA: Carousel House; 1984.

57. Shah AP. Comprehensive Assessment of Accentedness and Intelligibility (CAAI). Cleveland, OH: EBAM Institute, LLC; 2007.

58. Xu Y, Bolstad AL, Shen J, et al. Speak for success: a pilot intervention study on communication competence of post-hire international nurses. J Nurs Regul. 2010;1 (2):42-48. doi: 10.1016/S2155-8256(15)30350-1.

59. Australian Medical Council Clinical Examination. https://www.amc.org.au/assessment/clinical-exam. Accessed November 21, 2019.

60. Compton AJ. Compton Phonological Assessment of Foreign Accent. San Francisco, CA: Carousel House; 1983.

61. Dato D. Psycholinguistic Aspects of Foreign Accent. Rockville, MD: Bilingual Communications Institute; 1996.
62. Sikorski LD. POEC - S, Electronic Edition (Proficiency in Oral English Communication - Screen). 3rd ed. Santa Ana, CA: LDS \& Associates, LLC; 2005.

63. McCroskey JC. An Introduction to Rhetorical Communication. 4th ed. Englewood Cliffs, NJ: Prentice-Hall; 1982.

64. Rosenberg M. Society and the Adolescent Self-Image (rev. ed.). Middletown, CT: Wesleyan University Press; 1989.

65. McCroskey JC, McCroskey LL. Self-report as an approach to measuring communication competence. Commun Res Rep. 1988;5(2):108-113.

66. Sikorski LD. Regional accents: a rationale for intervening and competencies required. Semin Speech Lang. 2005 May;26(2):118-125. doi: 10.1055/s-2005-871207.

67. Baugh J. Linguistic Profiling and Discrimination. In: García O, Flores N, Spotti M, eds. The Oxford Handbook of Language and Society. Oxford, England: Oxford University Press; 2017.

68. Flege JE, MacKay IRA. Perceiving vowels in a second language. Stud Second Lang Acquis. 2004 Mar;26(1):1-34. doi: $10.1017 /$ S0272263104026117.

69. Lengeris A, Hazan V. The effect of native vowel processing ability and frequency discrimination acuity on the phonetic training of English vowels for native speakers of Greek. J Acoust Soc Am. 2010 Dec;128(6):3757-3768. doi: 10.1121/1.3506351.

70. Franklin AD, Stoel-Gammon C. Using multiple measures to document change in English vowels produced by Japanese, Korean, and Spanish speakers: the case for goodness and intelligibility. Am J Speech Lang Pathol. 2014 Nov;23(4):625-640. doi: 10.1044/2014_AJSLP-13-0144.

71. Flege JE, Munro MJ, Mackay IRA. Effects of age of second-language learning on the production of English consonants. Speech Commun. 1995 Jan;16(1):1-26. doi: 10.1016/0167-6393(94)00044-B.

72. Sheldon A, Strange W. The acquisition of / $/$ / and /l/ by Japanese learners of English: evidence that speech production can precede speech perception. Appl Psycholinguist. 1982 Sep;3(3):243-261. doi: 10.1017/S0142716400001417.

73. Derwing T, Rossiter MJ. The effects of pronunciation instruction on the accuracy, fluency, and complexity of L2 accented speech. Appl Lang Learn. 2003 Jan;13(1):1-17.

74. Derwing TM, Munro MJ, Wiebe G. Evidence in favor of a broad framework for pronunciation instruction. Lang Learn. 1998 Dec;48(3):393-410. doi: 10.1111/0023-8333.00047.

75. Behrman A, Ferguson SH, Akhund A, Moeyaert M. The effect of clear speech on temporal metrics of rhythm in Spanish-accented speakers of English. Lang Speech. 2019 Mar;62(1):5-29. doi: 10.1177/0023830917737109.

76. Strange W, Dittmann S. Effects of discrimination training on the perception of $/ \mathrm{r}-\mathrm{l} /$ by Japanese adults learning English. Percept Psychophys. 1984 Aug;36(2):131-145.

77. Clarke CM, Garrett MF. Rapid adaptation to foreign-accented English. J Acoust Soc Am. 2004 Dec;116(6):3647-3658. doi: $10.1121 / 1.1815131$.

78. Dansereau DF, Joe GW, Dees SM, Simpson DD. Ethnicity and the effects of mapping-enhanced drug abuse counseling. Addict Behav. 1996 May-Jun;21(3):363-376.

This article meets the Accreditation Council for Graduate Medical Education and the American Board of Medical Specialties Maintenance of Certification competencies for Patient Care and Interpersonal and Communication Skills.

C2019 by the author(s); licensee Ochsner Journal, Ochsner Clinic Foundation, New Orleans, LA. This article is an open access article distributed under the terms and conditions of the Creative Commons Attribution (CC BY) license (creativecommons.org/licenses/by/4.0/legalcode) that permits unrestricted use, distribution, and reproduction in any medium, provided the original author(s) and source are credited. 